\title{
Software Implemented Fault Tolerance in Hypercube
}

\author{
D.R. Avresky ${ }^{1}$ and S. Geoghegan ${ }^{2}$ \\ 1 Dept. of Electrical and Computer Eng., Boston University \\ 8 Saint Mary's Street, Boston, MA 02215 \\ avresky@bu . edu \\ 2 Computer Science Dept., Texas A\&M University \\ College Station, TX 77840
}

\begin{abstract}
This paper presents Software Implemented Fault Tolerance 1 (SIFT) for hypercubes which is implemented by means of a software layer. It is written in each node of the nCube parallel computer. The SIFT utilizes an error detection application software and fast reconfiguration algorithm for avoiding faulty nodes. The Balance Spanning Tree (BST) is used for embedding tree-based algorithms into the hypercube topology. Any single faulty node in the hypercube can be tolerated by the software layer. More than $90 \%$ of the multiple faults can be tolerated without backtracking. The SIFT approach has been successfully implemented for a quadtree data compression algorithm for $64 \times 64,128 \times 128$ compressible and uncompressible data. The experiments were run on 4 and 16 node nCubes. The time overhead (reconfiguration and recomputation time) incurred by the injected fault was experimentally estimated. The coverage factor, provided by the error-detection software, has been estimated by means of nSOFIT for the quadtree data compression algorithm.
\end{abstract}

\section{Introduction}

This paper presents Software Implemented Fault Tolerance (SIFT) for hypercubes which is implemented by means of a software layer, written in each node of the nCube parallel computer, with error-detection application software and fast reconfiguration algorithm for avoiding faulty nodes. The Balance Spanning Tree (BST) is used for embedding tree-based algorithms into the hypercube topology. A spanning tree is a connected graph that spans the nodes of the graph, forming a tree with no cycles [3].

Spanning trees have been shown to be useful in broadcasting and multicasting algorithms [4]. They also have been used in optimal communications and personalized communication in hypercubes [4]. Reconfiguration of faulty hypercubes using GST, BST and CUST are presented in [1]. The Balance Spanning Tree (BST) is used for embedding tree-based algorithms into the hypercube topology.

$\overline{1}$ This work was funded by National Science Foundation Grant MIP-963096

P. Amestoy et al. (Eds.): Euro-Par'99, LNCS 1685, pp. 515 5181999.

(C) Springer-Verlag Berlin Heidelberg 1999 
Any single-node fault in the hypercube can be tolerated by the software layer. More than $90 \%$ of the multiple faults can be tolerated without backtracking. The faults in the application level in each node are injected by means of the parallel version of the Software Fault Injection Tool (nSOFIT) for nCube. The software based fault injection tools are widely used for validating the error detection software and mechanisms [2]. The time overhead (reconfiguration and recomputation time) incurred by the injected fault is experimentally evaluated. The proposed algorithm is scalable, i.e., by increasing the size of the hypercube the reconfiguration time is constant and the recomputation time is decreasing. For reconfiguration and recovery, we make the assumptions, which are typical ones related to fault tolerance of hypercubes [1].

\section{Balanced Spanning Tree (BST) and Data Distribution}

The reconfiguration algorithm for a BST is based on finding a new path that avoids the faulty node. We have the same assumptions, and if any node $f$ is detected as faulty, the reconfiguration process takes place. The spanning tree is reconstructed by connecting the children of $f$ to the parent of $f$ via one unused link and another link that might have been used before failure.

In [1], the new parent was computed as $v_{n e w}=v_{c} \oplus v_{f} \oplus v_{p}$ where $v_{f}$ is the faulty node, $v_{c}$ is a child of $v_{f}, v_{p}$ is the parent of $v_{f}$ and $v_{n e w}$ is the replacement for $v_{f}$. The path $v_{c} v_{n e w} v_{p}$ is then used to replace the path $v_{c} v_{f} v_{p}$. It's been proven the large portion of the multiple faults and any single faulty link can be tolerated [1].

Construction of a complete reconfiguration algorithm must consider some data distribution and result collection factors. The typical algorithm using the BST involves three phases. First, the data is distributed to all the nodes in the BST. Second, all the nodes process their respective parts of the data. Finally, each node sends its results up to the root of the BST. During any of these steps, a node may fail and the parent and children nodes must invoke the reconfiguration algorithm to tolerate the fault. Thus, the algorithm must ensure that the input data is properly distributed to all the nodes, the data assigned to the faulty node are processed by an alternate node, and the results are collected at the root of the BST. The data must be distributed in such a way that all the nodes receive the data that is to be processed on that node. Following the failure of a node, all the fault-free nodes must still receive the appropriate data. In addition, an alternate node must have access to the data assigned to the faulty node in order to process the data in place of the faulty node. To meet these criteria, the distribution is achieved by first partitioning the data and assigning one partition to each BST node. All partitions are then sent to the root of the BST. Next, the root sends each of its children all the partitions assigned to the nodes in the subtree rooted by that child. This process continues until all the nodes receive the appropriate data. This distribution algorithm ensures that all the ancestors of each node, i, contain the data assigned to node i. 


\section{Quadtree Data Compression Algorithm}

The SIFT approach for hypercube topology, using BST, is suitable for divideand-conquer, sorting and similar tree based programs. A quadtree can be used to store and/or compress two-dimensional arrays or graphical regions such as those used for image processing [5]. To validate the SIFT approach, the quadtree data compression algorithm was written and executed on a BST embedded in a hypercube topology. Three separate test were run to measure different parameters of the quadtree algorithm. First, the overhead incurred by the reconfiguration step was measured. Next, the overhead incurred by the different fault-tolerance methods was measured. Finally, the percentage of fault detected achieved by the fault tolerance methods was estimated. The time required to execute the reconfiguration algorithm on each node has been measured and is presented in Table 1. Two versions of this experiment were run. In the first test, a 4-node BST was used in which $v_{f}=1, v_{c}=3, v_{p}=0$, and $v_{\text {new }}=2$. The second experiment involved a 16-node BST in which $v_{f}=7, v_{c}=15, v_{p}=3$, and $v_{\text {new }}=11$. In each experiment, a timer was placed in the code involved in the reconfiguration process. As the data compression program was executing, $v_{f}$ was forced to fail by fault injection, using nSOFIT. Three different time overhead values are obtained since each of the three nodes involved in the reconfiguration process performs a different task. As seen by the values in the table, the overhead incurred in each node is very small and does not vary with the size of the BST.

\begin{tabular}{|c|c|c|}
\hline Node & $\begin{array}{c}\text { Overhead for 4 nodes } \\
\text { (seconds) }\end{array}$ & $\begin{array}{c}\text { Overhead for 16 nodes } \\
\text { (seconds) }\end{array}$ \\
\hline$v_{c}$ & 0.0005 & 0.0004 \\
\hline$v_{p}$ & 0.0006 & 0.0006 \\
\hline$v_{\text {new }}$ & 0.0004 & 0.0004 \\
\hline
\end{tabular}

Table 1. Reconfiguration overhead and parameters

The results of different experiments are summarized in Table 2 . The speedup in Table 2 is the ratio of the time it takes to run the quadtree algorithm on 16 nodes vs. the execution time on 4 nodes system.

The tool(SOFIT) [2] is designed to evaluate the coverage factor of the implemented error-detection technique by applying a software approach to injecting faults in a computer system while a user application is executing. In this experiment, faults of 10 cycles duration were injected into the address bus, CPU, data bus, and memory. The coverage factor was determined using SOFIT to inject faults into node 7 as the quadtree algorithm was executed on a 16 node hypercube array. The results are summarized in Table 3 These results have been used to estimate the coverage factor, provided by the duplex error- detection scheme. 


\begin{tabular}{|c|c|c|c|c|c|c|}
\hline $\begin{array}{l}\text { Nodes } \\
\text { (number) }\end{array}$ & $\begin{array}{l}\text { Simplex } \\
\text { w/out "I'm } \\
\text { Alive" }\end{array}$ & $\begin{array}{l}\text { Simplex } \\
\text { with "I'm } \\
\text { Alive" }\end{array}$ & $\begin{array}{l}\text { Duplex } \\
\text { w/out } \\
\text { "I'm } \\
\text { Alive" }\end{array}$ & $\begin{array}{l}\text { Duplex } \\
\text { with "I'm } \\
\text { Alive" }\end{array}$ & $\begin{array}{l}\text { Time for } \\
\text { Duplex } \\
\text { with "I'm } \\
\text { Alive" } \\
\text { reconf. and } \\
\text { recomp. } \\
\text { (s) }\end{array}$ & $\begin{array}{l}\text { Recomp. } \\
\text { overhead } \\
\text { on vp } \\
(0,3)(\mathrm{s})\end{array}$ \\
\hline \multicolumn{7}{|c|}{ Uncompressible $64 \times 64$ data } \\
\hline 4 & 0.0759 & 0.0771 & 0.2065 & 0.2095 & 0.2109 & 0.0760 \\
\hline 16 & 0.0363 & 0.0380 & 0.1252 & 0.1284 & 0.1299 & 0.0191 \\
\hline speedup & 2.09 & 2.02 & 1.649 & 1.6316 & 1.62 & 3.97 \\
\hline \multicolumn{7}{|c|}{ Compressible $64 \times 64$ data } \\
\hline 4 & 0.0503 & 0.0515 & 0.1257 & 0.1283 & 0.1297 & 0.0877 \\
\hline 16 & 0.0292 & 0.0309 & 0.0681 & 0.0706 & 0.0721 & 0.0221 \\
\hline speeedup & 1.72 & 1.66 & 1.8458 & 1.8172 & 1.798 & 3.968 \\
\hline \multicolumn{7}{|c|}{ Compressible $128 \times 128$ data } \\
\hline 4 & 0.7417 & 0.7439 & 2.0905 & 2.2084 & 2.2097 & 0.7786 \\
\hline 16 & 0.0653 & 0.0671 & 0.2139 & 0.2192 & 0.2207 & 0.00877 \\
\hline speedup & 11 & 11.09 & 9.77 & 10.07 & 10.01 & 8.88 \\
\hline
\end{tabular}

Table 2. Duration for quadtree algorithm

\begin{tabular}{|l|r|r|r|r|r|r|r|r|r|r|}
\hline & OS Detected & FTAM Detected & Timeout & \multicolumn{2}{|c|}{ Incorrect Result } & \multicolumn{2}{|c|}{ Correct Result } \\
\hline Address bus & 125 & $83.33 \%$ & 25 & $16.67 \%$ & $0.00 \%$ & 0 & $0.00 \%$ & 150 & $100.00 \%$ \\
\hline Data bus & 17 & $11.33 \%$ & 108 & $72.00 \%$ & 2 & $1.33 \%$ & 23 & $15.33 \%$ & 127 & $84.67 \%$ \\
\hline Memory & 109 & $72.67 \%$ & 26 & $17.33 \%$ & 2 & $1.33 \%$ & 13 & $8.67 \%$ & 137 & $91.33 \%$ \\
\hline CPU & 145 & $96.67 \%$ & 5 & $3.33 \%$ & 0 & $0.00 \%$ & 0 & $0.00 \%$ & 150 & $100.00 \%$ \\
\hline
\end{tabular}

Table 3. Coverage for the quadtree algorithm

\section{References}

[1] D. R. Avresky, "Embedding and Reconfiguration of Spanning Trees in Faulty Hypercube," IEEE Transaction on Parallel and Distributed Systems, vol 10, no.3, March 1999, USA.

[2] D. R. Avresky, S. J. Geoghegan, and P. K. Tapadiya, "A Software-Based Fault Injecton Tool", International Journal of Computer Systems Science and Engineering, vol 13, no. 6. pp. 125-135. November 1998.

[3] T. Cormen, C. Leiserson, and R. Rivest, Introduction to Algorithms, McGraw-Hill, pp. 498-513, 1990.

[4] S. Johnsson, and C. Ho, "Optimum Broadcasting and Personalized Communication in Hypercubes," IEEE Trans. on Comp., Vol. C-38, No. 9, pp. 197-202, Sept. 1989.

[5] H. Samet, , "The Quadtree and related Hierarchial Data Structures ," ACM Comp. Surveys, 16:2, pp 187-260 1984. 